20

\title{
Оптимальное управление внутривенной лазерной абляцией
}

\author{
(C) А.Е. Ковтанюк ${ }^{1,2}$, А.Ю. Чеботарев ${ }^{1,2}$, А.А. Астраханцева ${ }^{1}$, А.А. Сущенко ${ }^{1,2}$ \\ ${ }^{1}$ Дальневосточный федеральный университет, \\ 690950 Владивосток, Россия \\ ${ }^{2}$ Институт прикладной математики Дальневосточного отделения РАН, \\ 690041 Владивосток, Россия \\ e-mail: kovtanyuk.ae@dvfu.ru
}

Поступила в редакцию 30.07.2019 г.

В окончательной редакции 01.06.2020 г.

Принята к публикации 05.06.2020 г.

На основе начально-краевой задачи для модели радиационно-кондуктивного теплообмена изучаются тепловые процессы, возникающие при внутривенной лазерной абляции. Ставится задача оптимального управления, заключающаяся в приближении решения начально-краевой задачи к заданному температурному профилю в определенной точке модельной области. В качестве управления берутся мощности источника, идущие на излучение и нагрев карбонизированного наконечника оптоволокна. Предложен и численно реализован итерационный алгоритм решения поставленной задачи.

Ключевые слова: внутривенная лазерная абляция, перенос излучения, кондуктивный перенос тепла, оптимальное управление.

DOI: $10.21883 /$ OS.2020.09.49882.242-20

\section{Введение}

В 1999 г. появилось первое сообщение о клиническом внутрисосудистом применении лазера с длиной волны $810 \mathrm{~nm}$ для осуществления внутривенной лазерной абляции (ВВЛА) [1]. При проведении ВВЛА лазерное оптоволокно вводится в поврежденную вену. Далее оптоволокно вытягивается из вены, при этом по нему передается лазерное излучение. Торец оптоволокна, из которого выходит излучение, обычно покрыт карбонизированным слоем, который разделяет поступающую энергию, в результате чего одна ее часть поглощается карбонизированным слоем, что вызывает его нагрев, другая часть идет на излучение. Тепло от нагретого карбонизированного слоя передается в кровь посредством кондуктивного теплообмена. Теплообмен многократно усиливается за счет потока пузырьков, образующихся на разогретом торце оптоволокна. Излучение, поступающее в кровь и окружающую ткань, частично поглощается, что сопровождается выделением тепла. В итоге образованная и поступившая за счет различных механизмов тепловая энергия вызывает значительный нагрев вены, что приводит к ее облитерации (закрытию сосуда). Поскольку большинство побочных эффектов при проведении ВВЛА незначительны, а серьезные осложнения редки, лазерная абляция представляет собой безопасную и минимально инвазивную процедуру, обладающую высокой эффективностью в лечении варикозной болезни [2].

Другим популярным подходом, обладающим малой инвазивностью и высокой эффективностью, является радиочастотная абляция вен (РЧА). В работе [3] на основе обзора различных исследований проводится сравнение лазерной и радиочастотной абляции по пяти критериям, включающим эффективность, побочные эффекты, серьезные осложнения, рецидив и качество жизни. Отмечается, что лазерная абляция обладает несколько большей эффективностью и меньшей частотой рецидивов по сравнению с РЧА. Например, результаты мета-анализа данных 4876 пациентов после лазерной и 2514 после радиочастотной абляции демонстрируют следующие показатели успешно выполненной облитерации, которые составляют 92.9\% для ВВЛА и $88.8 \%$ для РЧА. Серьезные осложнения редко встречаются в обоих случаях. Незначительные осложнения, такие как послеоперационная боль и гематомы, более характерны для ВВЛА. Вероятную причину, по которой ВВЛА приводит к большей послеоперационной боли, чем РЧА, объясняют результаты экспериментов [4], согласно которым лазерная абляция характеризуется более высокой пиковой температурой, хотя и в течение короткого промежутка времени, тогда как у радиочастотной абляции более низкие, хотя и более продолжительные, максимальные температуры.

Оптимизация параметров излучения при проведении ВВЛА позволяет осуществить успешную облитерацию вены с минимальной частотой и тяжестью осложнений. То есть оптимальное излучение, с одной стороны, должно обеспечивать достаточно высокую температуру внутри вены для осуществления облитерации, с другой стороны, сгенерированное температурное поле должно быть относительно безопасным для окружающей вену ткани. Основными параметрами, влияющими на эффективность и безопасность процедуры лазерной абляции, являются мощность лазера, длина волны лазерного излучения, скорость движения оптоволокна, соотношение до- 
лей мощности лазера, идущих на излучение и на нагрев наконечника (карбонизированного слоя) оптоволокна. Как правило, при проведении лазерной абляции используется лазер с длиной волны от 810 до $1470 \mathrm{~nm}$. Достаточно распространенными являются диапазоны скорости движения оптоволокна от 1 до $3 \mathrm{~mm} / \mathrm{s}$ и мощности лазерного излучения от 10 до $15 \mathrm{~W}$.

Математическое моделирование радиационных и тепловых процессов, возникающих при проведении ВВЛА, является весьма актуальным в контексте определения оптимальных параметров излучения, обеспечивающих заданное распределение температуры внутри вены и в перивенозной ткани. Классическая модель, описывающая взаимодействие излучения оптического диапазона с биотканью, включает уравнение переноса излучения и уравнение теплопроводности [5]. Соответственно, расчет радиационных и температурных полей включает в себя нахождение решения уравнения переноса, вычисление по нему объемной плотности поглощенной энергии излучения и последующее нахождение решения уравнения теплопроводности, в которое поглощенная энергия излучения входит в качестве источников тепла. Нередко при решении различных задач переноса излучения учитываются эффекты отражения и преломления на границах раздела сред с различными показателями преломления [6,7], что достаточно хорошо моделируется методом Монте-Карло [7,8]. С целью упрощения модели уравнение переноса излучения иногда заменяется на его диффузионное приближение, которое также позволяет описывать эффекты отражения и преломления. В дополнении к этому диффузионная модель может учитывать тепловое излучение, индуцированное самой средой, что приводит к нелинейной модели сложного теплообмена $[9,10]$. В работе [9] оценивается вклад внутреннего теплового излучения, а также эффектов отражения и преломления в профили температуры и поглощенной энергии излучения для кожного покрова, находящегося под воздействием солнечного излучения.

Основными эффектами, которые обычно учитываются при моделировании ВВЛА, являются кондуктивный теплообмен, перенос излучения и его поглощение с выделением тепла, а также перенос тепла потоком пузырьков, образующихся на наконечнике оптоволокна. В работе [11] приведены результаты экспериментов, демонстрирующих влияние движущихся пузырьков, образованных на наконечнике оптоволокна, на температурное поле. В работах [12-14] на основе оценки экспериментальных данных перенос тепла потоком пузырьков моделируется использованием кусочно-постоянного коэффициента теплопроводности, зависящего от температуры следующим образом: при достижении температуры в некоторой точке $95^{\circ} \mathrm{C}$ и более коэффициент теплопроводности увеличивается в 200 раз.

Результаты численного моделирования ВВЛА для длин волн непрерывного излучения в диапазоне от 810 до $1470 \mathrm{~nm}$ и различных диаметров вен приведены в работах [12-14]. В них, в частности, оценка эффективности лазерной абляции осуществляется через анализ температурных профилей в точках наблюдения, находящихся в различных зонах вычислительной области (кровь, внутренняя и внешняя стенки вены, перивенозная ткань). Отметим, что в указанных работах усредненная интенсивность излучения вычисляется по явной формуле, описывающей радиационное поле точечного источника в неограниченной однородной среде.

В работе [15] отмечается важность численного моделирования при выборе оптимальной мощности лазерного излучения для различных длин волн лазерного излучения и различных диаметров вен. Авторы проводят сравнительный численный анализ эффективности ВВЛА в случае непрерывного и импульсного (с продолжительностью импульса от 1 до $3 \mathrm{~s}$ ) излучения с длиной волны 810 и $980 \mathrm{~nm}$. Отмечается эффективность обоих подходов. И хотя импульсное излучение обеспечивает несколько меньший нагрев ткани, его использование требует точного позиционирования волокна и большую продолжительность операции. В итоге авторы отмечают, что многие врачи предпочитают выполнять лазерную абляцию, используя непрерывный источник излучения.

Сложности использования импульсного излучения при проведении ВВЛА, отмеченные в работе [15], можно избежать, если значительно уменьшить продолжительность импульса. В работе [16] проводится численный анализ эффективности ВВЛА при использовании импульсного излучения с длиной волны $1320 \mathrm{~nm}$, при продолжительности импульса $1.2 \mathrm{~ms}$ и частоте импульсов 30-50 Hz. Проводится сравнение со случаем непрерывного излучения с длиной волны $980 \mathrm{~nm}$. Отмечается, что при использовании импульсного излучения можно достичь тот же эффект, как и при непрерывном излучении, используя меньшую суммарную мощность. Сопоставляя результаты проведенного численного моделирования с опубликованными статистическими данными, авторы отмечают, что оба подхода являются достаточно эффективными и имеют сходные побочные эффекты.

Отметим, что в работах [12-16] при выборе оптимальных параметров излучения используется прямое многократное моделирование, осуществляющее по сути перебор различных вариантов с целью поиска некоего оптимального решения. Данный подход не является эффективным. К тому же остаются без ответа вопросы, связанные с существованием и единственностью оптимального решения. В настоящей работе предлагается принципиально другой подход в поиске оптимальных параметров излучения, а именно путем решения задачи оптимального управления. Следуя работам [12-14], в которых эффективность ВВЛА оценивается по поведению температурных профилей, авторами рассматривается задача оптимального управления ВВЛА, заключающаяся в подборе параметров излучения с целью приблизить температурный профиль в определенной точке модельной области к заданному графику, характеристики которого (максимальная температура и продолжительность 


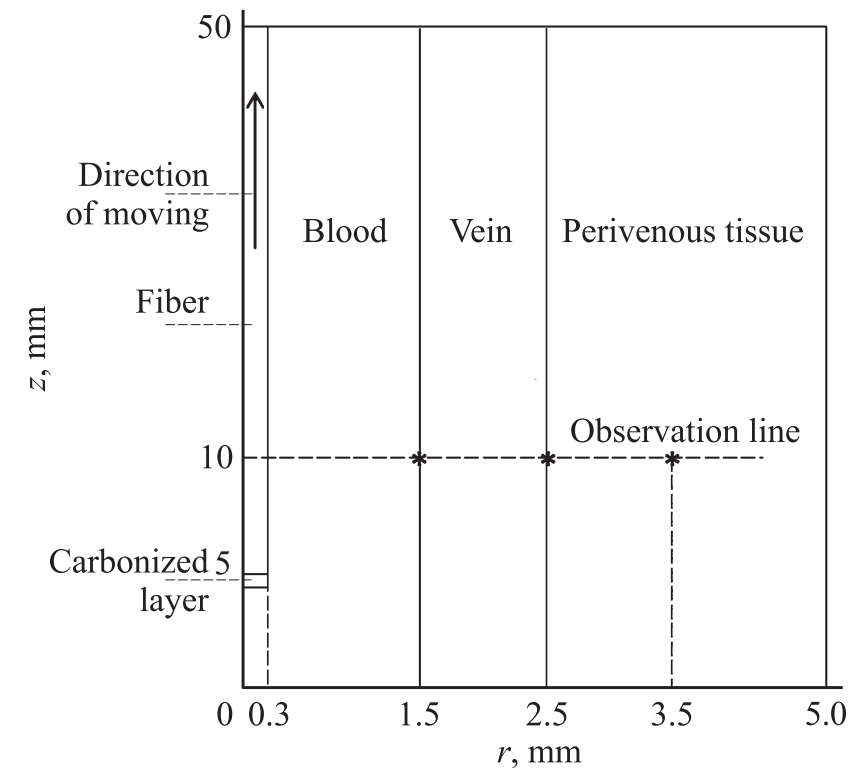

Рис. 1. Схема расчетной области в цилиндрических координатах при угловой симметрии. На рисунке отмечены точки наблюдения и начальное положение оптоволокна. Толщина карбонизированного слоя $0.2 \mathrm{~mm}$.

кипения) обеспечивают успешное проведение процедуры ВВЛА. В качестве управления процессом берутся мощности источника, идущие на излучение и нагрев наконечника оптоволокна. Ввиду нелинейности модели, вызванной зависимостью коэффициента теплопроводности от температуры, для решения задачи оптимального управления используется итерационный алгоритм, на каждом шаге которого для нахождения нового приближения решается начально-краевая задача. Численная реализация алгоритма осуществляется на основе метода конечных элементов для случая непрерывного излучения с длиной волны $810 \mathrm{~nm}$. Проведены эксперименты по выбору оптимальных параметров излучения, обеспечивающих приближение температурного профиля к заданному графику в точке, находящейся на внутренней стенке вены.

\section{Математическая модель}

В качестве математической модели, описывающей тепловые и радиационные процессы в биологической ткани, находящейся под воздействием лазерного излучения, рассмотрим систему уравнений, включающую уравнение теплопроводности и диффузионное приближение уравнения переноса излучения.

Перенос тепла и излучения будем рассматривать в среде, состоящей из четырех подобластей, включающих кровь, стенки вены, перивенозную ткань и оптоволокно. Схема расчетной области $G$ с границей $\Gamma$ в цилиндрической системе координат, при угловой симметрии, приведена на рис. 1 (линейные размеры в миллиметрах).
В работах [12-16] при моделировании ВВЛА используется уравнение теплопроводности, учитывающее поступление тепла от энергии поглощенного излучения. Однако в указанных работах, интенсивность излучения рассчитывается на основе явной формулы решения диффузионного уравнения для случая бесконечной однородной среды с точечным источником излучения. В отличие от указанных работ будем применять диффузионное приближение уравнения переноса излучения, учитывающее многокомпонентную структуру среды. Поскольку излучение попадает в среду через торец оптоволокна, источник излучения можно описывать как поверхностный, задавая его через соответствующее граничное условие, либо как объемный, отожествляя его с карбонизированным наконечником. Останавливаясь на случае объемного источника излучения, запишем систему дифференциальных уравнений, описывающих радиационные и тепловые процессы при проведении ВВЛА, в следующем виде:

$$
\begin{gathered}
-\nabla \cdot(\alpha \nabla \varphi)+\mu_{a} \varphi=P_{\varphi} \chi V^{-1}, \\
\rho c_{p} \frac{\partial \theta}{\partial t}-\nabla \cdot(k \nabla \theta)=\mu_{a} \varphi+P_{\theta} \chi V^{-1} .
\end{gathered}
$$

Здесь $\varphi(x, t)$ - усредненная по направлениям интенсивность излучения, $\theta(x, t)$ - температура, $\alpha(x)-$ коэффициент диффузии фотонов, $\alpha=1 / 3\left(\mu_{s}^{\prime}+\mu_{a}\right), \mu_{a}(x)-$ коэффициент поглощения, $\mu_{s}^{\prime}-$ приведенный коэффициент рассеяния, $k(x, t)$ - коэффициент теплопроводности, $c_{p}(x, t)$ - удельная теплоемкость, $\rho(x, t)-$ плотность, $V$ - объем наконечника (карбонизированного слоя) оптоволокна, $P_{\varphi}-$ мощность источника, идущая на излучение, $P_{\theta}-$ мощность источника, идущая на нагрев наконечника оптоволокна, $P_{\varphi}, P_{\theta} \geq 0$, $P_{\varphi}+P_{\theta} \leq P_{\max }, P_{\max }-$ максимальная мощность источника, $\chi(x, t)$ - характеристическая функция части среды, в которой находится наконечник оптоволокна, $x \in G, t \in(0, T)$. Диффузионное уравнение переноса излучения (1) описывает распространение излучения от движущегося источника и учитывает эффекты поглощения и рассеяния фотонов. Уравнение теплопроводности (2) описывает кондуктивный перенос тепла с учетом тепла, поступающего от энергии поглощенного излучения $\left(\mu_{a} \varphi\right)$, и тепла, выделяемого нагретым наконечником оптоволокна $\left(P_{\theta} \chi V^{-1}\right)$.

Уравнения (1),(2) дополняются следующими условиями на границе Г и в начальный момент времени:

$$
\begin{gathered}
\alpha \partial_{n} \varphi+\left.\beta \varphi\right|_{\Gamma}=0, \\
k \partial_{n} \theta+\left.\gamma\left(\theta-\theta_{b}\right)\right|_{\Gamma}=0, \\
\theta(x, 0)=\theta_{0} .
\end{gathered}
$$

Условие (3) представляет собой популярное граничное условие Маршака [17]. С учетом цилиндрической системы координат параметры $\beta$ и $\gamma$ равны нулю при $r=0$ (рис. 1). На остальных границах $\beta=0.5, \gamma=1$. На границах раздела сред ставятся стандартные условия непрерывности решения и равенства тепловых и 
радиационных потоков. В итоге радиационные и тепловые процессы, протекающие в модельной области при выполнении ВВЛА, будут описываться решением начально-краевой задачи (1)-(5).

Следуя [12-14], моделирование переноса тепла потоком пузырьков, исходящих от наконечника оптоволокна, будем осуществлять, используя следующее представление для коэффициента теплопроводности крови:

$$
k(x, t)= \begin{cases}k_{0}, & \text { если } \theta(x, t)<95, \\ 200 k_{0}, & \text { если } \theta(x, t) \geq 95,\end{cases}
$$

где $k_{0}$ - значение коэффициента теплопроводности крови, соответствующее температуре в начальный момент времени.

При проведении расчетов на основе $(1)-(5)$ используются оптические (для длины волны $810 \mathrm{~nm}$ ) и теплофизические параметры задачи, представленные в таблице. Величины $\theta_{b}$ и $\theta_{0}$ равны 37 . Во всех расчетах начальное положение конца оптоволокна соответствует $z=5$, и его скорость движения равна $2 \mathrm{~mm} / \mathrm{s}$.

В настоящей работе объектом численного моделирования является ВВЛА, проводимая с использованием непрерывного лазерного излучения (излучение с постоянной мощностью). Тем не менее система (1)-(5) может также применяться для моделирования радиационных и тепловых полей, индуцированных импульсным излучением. В этом случае параметры $P_{\varphi}$ и $P_{\theta}$ зависят от времени. Однако численная реализация задачи в случае импульсного излучения может сопровождаться значительными вычислительными затратами. Так, по оценке авторов, численная реализация задачи (1)-(5) в случае импульсного источника, рассмотренного в [16], с продолжительностью импульса $1.2 \mathrm{~ms}$, потребует минимум в 10 раз больше машинного времени, чем в случае непрерывного источника.

Об эффективности лазерной абляции можно судить по поведению температурных профилей в различных точках расчетной области [12-14]. Анализ температурных профилей позволяет оценить является ли величина и продолжительность нагрева внутри вены достаточными для осуществления облитерации, и в тоже время, будет ли тепловое воздействие вне вены безопасным для живой ткани. На рис. 2 демонстрируется поведение температурных профилей в трех точках наблюдения $(1.5,10)$, $(2.5,10)$ и $(3.5,10)$ при излучении со следующим распределением мощности источника: $\left(P_{\varphi}, P_{\theta}\right)=(7,3)$ (здесь и далее мощность дается в Ваттах). Точки наблюдения изображены на рис. 1 и находятся на внутренней стенке вены, на внешней стенке вены и в перивенозной ткани. Как видно из графиков, несмотря на высокую температуру внутри вены, температура в перивенозной ткани является вполне безопасной.

Сравним модель (1)-(5) с ее упрощенным аналогом, использованном в $[12-16]$, в котором интенсивность излучения вычисляется по следующей формуле, справедливой для точечного источника с координатами $\left(0, z_{0}\right)$ в

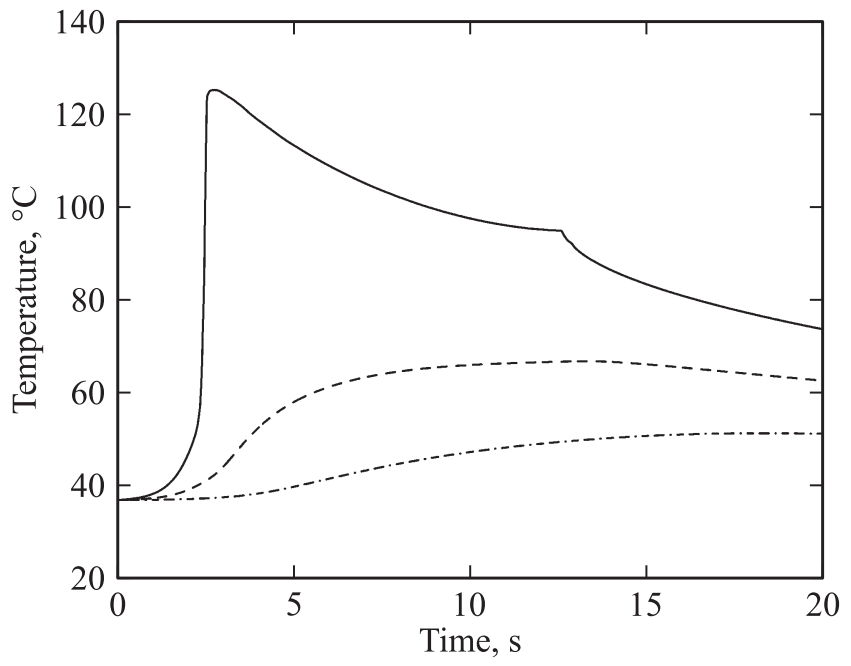

Рис. 2. Температурные профили в точках: $(1.5,10)$ - внутренняя стенка вены (сплошная линия); $(2.5,10)$ - внешняя стенка вены (штриховая линия); $(3.5,10)$ - перивенозная ткань (штрих-пунктирная линия).

бесконечной однородной среде:

$$
\varphi(r, z)=\frac{P_{\varphi} \exp \left(-\mu_{e} d(r, z)\right)}{4 \pi \alpha d(r, z)},
$$

где $\mu_{e}=\sqrt{\mu_{a} / \alpha}$ и функция $d(r, z)$ описывает расстояние между точками $\left(0, z_{0}\right)$ и $(r, z)$. В этом случае температурное поле, возникающее при ВВЛА, описывается моделью (2), (4), (5), (7). Температурные профили, полученные на основе модели (1)-(5) и модели (2), (4), (5), (7), представлены на рис. 3. Несмотря на некоторое различие графиков, можно сделать вывод, что с точки зрения практического применения упрощенная модель (2), (4), (5), (7) обеспечивает достаточно хорошее приближение к решению задачи (1)-(5). При удалении от источника излучения температурные профили становятся ближе. Это объясняется значительным уменышением в этих точках уровня излучения, и соответственно уменьшением вклада от энергии поглощенного излучения в температуру.

\section{Задача оптимального управления}

Будем оценивать эффективность лазерной абляции по поведению температурного профиля в выбранной точке расчетной области. Поставим задачу приблизить температурный профиль, являющийся решением задачи (1)-(5), к некоторому заданному графику, характеристики которого (например, максимальная температура и продолжительность кипения) обеспечивают успешное проведение процедуры ВВЛА. В качестве управления возьмем параметры $P_{\varphi}$ и $P_{\theta}$. Скорость движения оптоволокна и длина волны лазерного излучения являются заданными. 
Оптические (для длины волны $810 \mathrm{~nm}$ ) и термофизические параметры $[12,13]$

\begin{tabular}{l|c|c|c|c|c}
\hline \multicolumn{1}{c|}{ Части среды } & $\mu_{a}, \mathrm{~mm}^{-1}$ & $\mu_{s}^{\prime}, \mathrm{mm}^{-1}$ & $k, \mathrm{~W} /(\mathrm{mK})$ & $\rho, \mathrm{kg} / \mathrm{m}^{3}$ & $c_{p}, \mathrm{~J} /(\mathrm{kg} \mathrm{K})$ \\
\hline Кровь & 0.21 & 0.73 & 0.6 & 1000 & 4181 \\
Стенка вены & 0.2 & 2.4 & 0.56 & 1050 & 3780 \\
Перивенозная ткань & 0.017 & 1.2 & 0.56 & 1050 & 3780 \\
Оптоволокно & - & - & 1.3 & 2400 & 703
\end{tabular}

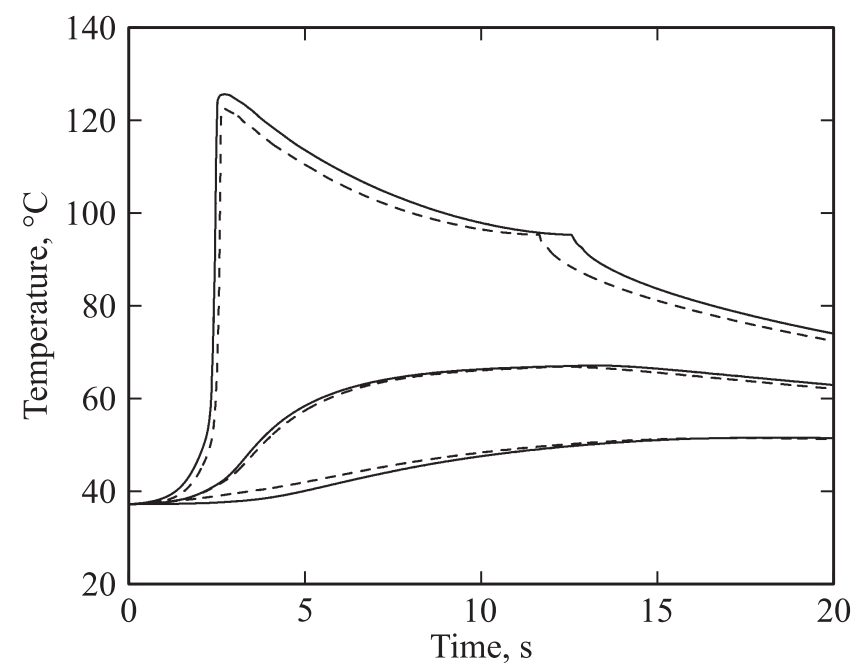

Pис. 3. Температурные профили в точках $(1.5,10),(2.5,10)$ и $(3.5,10)$ : сплошные линии - модель $(1)-(5)$; штриховые линии - упрощенная модель (2), (4), (5), (7), основанная на явной формуле интенсивности излучения точечного источника в однородной среде.

Формализуем постановку задачи оптимального управления. Пусть $x_{0}$ - точка наблюдения, в которой требуется обеспечить близость решения задачи (1)-(5) к заданному температурному профилю на промежутке времени $(0, T)$. Рассмотрим следующую функцию:

$$
J=\int_{0}^{T}\left(\theta\left(x_{0}, t\right)-\theta_{d}\left(x_{0}, t\right)\right)^{2} d t .
$$

Здесь $\theta_{d}$ - заданный температурный профиль.

Рассмотрим задачу оптимального управления, заключающуюся в минимизации функции $J$ за счет выбора пары $\left(P_{\varphi}, P_{\theta}\right)$, называемой управлением:

$$
J\left(P_{\varphi}, P_{\theta}\right) \rightarrow \min , \quad P_{\varphi}, P_{\theta} \geq 0, \quad P_{\varphi}+P_{\theta} \leq P_{\max } .
$$

Для решения задачи оптимального управления (9) разобьем задачу (1)-(5) на следующие три начальнокраевые задачи для пары функций $\varphi_{1}, \theta_{1}$ и функций $\theta_{2}, \theta_{3}$ :

$$
\begin{gathered}
-\nabla \cdot\left(\alpha \nabla \varphi_{1}\right)+\mu_{a} \varphi_{1}=\chi V^{-1}, \alpha \partial_{n} \varphi_{1}+\left.\beta \varphi_{1}\right|_{\Gamma}=0 \\
\rho c_{p} \frac{\partial \theta_{1}}{\partial t}-\nabla \cdot\left(k \nabla \theta_{1}\right)=\mu_{a} \varphi_{1},
\end{gathered}
$$

$$
\begin{gathered}
k \partial_{n} \theta_{1}+\left.\gamma \theta_{1}\right|_{\Gamma}=0, \theta_{1}(x, 0)=0 ; \\
\rho c_{p} \frac{\partial \theta_{2}}{\partial t}-\nabla \cdot\left(k \nabla \theta_{2}\right)=\chi V^{-1}, \\
k \partial_{n} \theta_{2}+\left.\gamma \theta_{2}\right|_{\Gamma}=0, \theta_{2}(x, 0)=0 ; \\
\rho c_{p} \frac{\partial \theta_{3}}{\partial t}-\nabla \cdot\left(k \nabla \theta_{3}\right)=0, \\
k \partial_{n} \theta_{3}+\left.\gamma\left(\theta_{3}-\theta_{b}\right)\right|_{\Gamma}=0, \theta_{3}(x, 0)=\theta_{0} .
\end{gathered}
$$

В итоге решение задачи (1)-(5) можно представить в виде

$$
\begin{gathered}
\theta=P_{\varphi} \theta_{1}+P_{\theta} \theta_{2}+\theta_{3}, \\
\varphi=\varphi_{1} P_{\varphi} .
\end{gathered}
$$

Подставим представление (14) в (8). В результате задача оптимального управления (9) сводится к минимизации функции

$$
\begin{gathered}
J_{0}\left(P_{\varphi}, P_{\theta}\right)=C_{1} P_{\varphi}^{2}+C_{2} P_{\theta}^{2}+2 C_{3} P_{\varphi} P_{\theta}+2 C_{4} P_{\varphi}+2 C_{5} P_{\theta}, \\
P_{\varphi}, P_{\theta} \geq 0, \quad P_{\varphi}+P_{\theta} \leq P_{\max },
\end{gathered}
$$

где

$$
\begin{gathered}
C_{1}=\int_{0}^{T} \theta_{1}^{2}(t) d t, \quad C_{2}=\int_{0}^{T} \theta_{2}^{2}(t) d t, C_{3}=\int_{0}^{T} \theta_{1}(t) \theta_{2}(t) d t \\
C_{4}=\int_{0}^{T} \theta_{1}(t)\left(\theta_{3}(t)-\theta_{d}(t)\right) d t \\
C_{5}=\int_{0}^{T} \theta_{2}(t)\left(\theta_{3}(t)-\theta_{d}(t)\right) d t .
\end{gathered}
$$

Здесь в выражениях для коэффициентов $C_{i}$, $i=1,2, \ldots, 5$ опущен аргумент $x_{0}$. Заметим, что

$$
J=J_{0}+\int_{0}^{T}\left(\theta_{3}\left(x_{0}, t\right)-\theta_{d}\left(x_{0}, t\right)\right)^{2} d t
$$

Таким образом, в случае, когда коэффициент теплопроводности не зависит от температуры, задача оптимального управления сводится к нахождению минимума квадратичной относительно переменных $P_{\varphi}, P_{\theta}$ функции $J_{0}$. Если минимум находится в треугольнике 
$P_{\varphi}, P_{\theta} \geq 0, P_{\varphi}+P_{\theta} \leq P_{\max }$, то для него справедливы следующие равенства:

$$
\begin{gathered}
P_{\varphi}=\left(C_{2} C_{4} C_{3}^{-1}-C_{5}\right)\left(C_{3}-C_{1} C_{2} C_{3}^{-1}\right)^{-1}, \\
P_{\theta}=-C_{1} C_{3}^{-1} P_{\varphi}-C_{4} C_{3}^{-1} .
\end{gathered}
$$

Если минимум квадратичной функции находится вне треугольника $P_{\varphi}, P_{\theta} \geq 0, P_{\varphi}+P_{\theta} \leq P_{\max }$, решение задачи (9) будет находиться на его границе, и для этого решения также можно получить равенства, связывающие оптимальную пару $P_{\varphi}, P_{\theta}$ с коэффициентами $C_{i}$, $i=1,2, \ldots, 5$.

Если учитывать зависимость коэффициента теплопроводности от температуры, например, используя представление (6), коэффициенты $C_{i}, i=1,2, \ldots, 5$ будут зависеть от $P_{\varphi}, P_{\theta}$. В этом случае для решения задачи оптимального управления нельзя напрямую воспользоваться формулами (15), (16). Возможным вариантом решения задачи является использование итерационной процедуры, которая будет представлена в следующем разделе.

Обозначим через параметр $\sigma$ черноту наконечника оптоволокна, которую определим как отношение мощности $P_{\theta}$ к суммарной мощности $P_{\varphi}+P_{\theta}$. Параметр $\sigma$ определяет долю энергии лазерного излучения, поглощаемую наконечником. В случае, когда требуется решить задачу оптимального управления при фиксированной черноте наконечника, управление осуществляется только суммарной мощностью $P_{\varphi}+P_{\theta}$. При фиксированном $\sigma$ отношение мощности $P_{\theta}$ к $P_{\varphi}$ будет также постоянно, обозначим эту величину за $\lambda$. Отметим, что

$$
\lambda=\frac{\sigma}{1-\sigma}, \quad \sigma \in[0,1)
$$

Тогда для нахождения оптимальной пары $\left(P_{\varphi}, P_{\theta}\right)$, минимизирующей функцию $J_{0}$, используются следующие формулы:

$$
\begin{gathered}
P_{\varphi}=-\left(C_{4}+\lambda C_{5}\right)\left(C_{1}+\lambda^{2} C_{2}+2 \lambda C_{3}\right)^{-1}, \\
P_{\theta}=\lambda P_{\varphi} .
\end{gathered}
$$

В случае, если учитывается зависимость коэффициента теплопроводности от температуры, нахождение оптимальной пары $\left(P_{\varphi}, P_{\theta}\right)$ осуществляется итерационно на основе формул (17) и (18).

Проанализируем влияние черноты наконечника на поведение температурного профиля при мощности излучения $10 \mathrm{~W}$. Наблюдение будем осуществлять в точке $(1.5,10)$, соответствующей внутренней стенке вены. Графики температурных профилей, соответствующие значениям черноты наконечника, равным 0,0.3 и 0.35, представлены на рис. 4. Как видно из графиков, при $\sigma=0$ имеет место значительное уменьшение уровня температуры по сравнению со случаями, когда $\sigma$ равно 0.3 и 0.35 . Также отметим, что различие в температурных профилях, соответствующих черноте наконечника 0.3 и 0.35 , с точки зрения практического

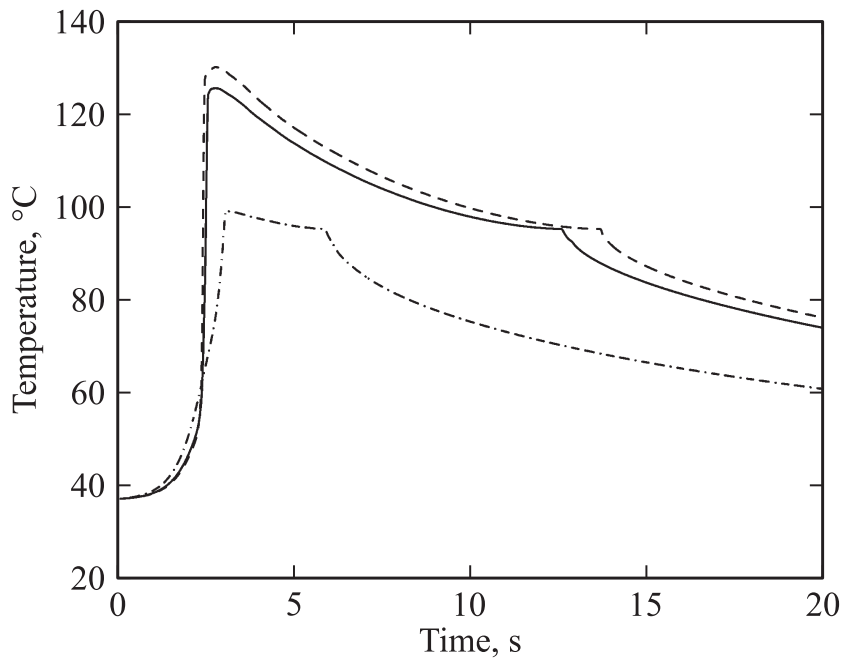

Рис. 4. Влияние черноты карбонизированного слоя $\sigma$ на поведение температурного профиля в точке $(1.5,10)$ при мощности излучения $10 \mathrm{~W}$ : штрихпунктирная линия $-\sigma=0$; сплошная линия $-\sigma=0.3$; штриховая линия $-\sigma=0.35$.

применения не является большим. Так, продолжительность „кипения“ в точке наблюдения (когда температура превышает $95^{\circ} \mathrm{C}$ ) увеличивается с 10 (при $\sigma=0.3$ ) до $11.2 \mathrm{~s}$ (при $\sigma=0.35$ ).

Роль карбонизированного наконечника оптоволокна в повышении эффективности лазерной термотерапии отмечается многими авторами [11-14,18-23]. Карбонизация может быть как естественной, т.е. результатом взаимодействия лазерного излучения с биологической средой $[11,18]$, так и искусственной, как результат контактного лазерного воздействия с различными материалами, например, с деревом [19,21], с активированным углем $[20,21]$, со смесью графита и силиконового лака технология SAC (strongly absorbing coating) [23].

Выбор той или иной технологии для создания карбонизированного покрытия определяет диапазон возможных значений черноты наконечника $\sigma$, который может быть обеспечен для проведения лазерной терапии. Так, покрытие, образованное непосредственным взаимодействием лазерного излучения с кровью, имеет черноту от 0.3 до 0.7 [18]. Карбонизация при контакте с деревом обеспечивает черноту от 0.1 до 0.2 [19]. Карбонизированный слой, получаемый с помощью технологии SAC, характеризуется чернотой в диапазоне от 0.3 до 0.5 [23]. Использование титаносодержащего конвертера с объемной долей диоксида титана 0.22 обеспечивает черноту наконечника 0.84 при длине волны $980 \mathrm{~nm}$ [22].

Карбонизированный наконечник оптоволокна нередко называется „hot tip“ [12-14], „blackened tip“ [19], также используется термин (углеродосодержащий) конвертер [21]. Толщина карбонизированного слоя определяется способом его создания. Так, в соответствии с результатами, представленными в работе [18], толщина карбонизированного слоя, образованного в результате 
взаимодействия лазерного излучения с кровью, составляет $0.02-0.03 \mathrm{~mm}$. В работе [21] приводится обзор различных конвертеров и их свойства. В частности, содержится классификация конвертеров, согласно которой они подразделяются на пленочные с толщиной карбонизированного слоя $0.05 \mathrm{~mm}$ и на объемные (плоские и сферические) с толщиной слоя до $0.15 \mathrm{~mm}$.

Отметим, что при проведении численных экспериментов авторами была выбрана толщина карбонизированного слоя $0.2 \mathrm{~mm}$, поскольку использование толщин меньше $0.1 \mathrm{~mm}$ сопряжено со значительными вычислительными затратами. Предварительно для обоснования этого выбора была изучена зависимость температурного поля от толщины наконечника. При проведении численного моделирования рассматривалась область $(0,2.5) \times(0,2)$, содержащая неподвижный наконечник толщиной 0.02 либо $0.2 \mathrm{~mm} \mathrm{c}$ центром в точке $(0,1)$. Сравнивались температурные профили, описывающие поперечные к стенке вены распределения температур, в различные моменты времени $(0.5,1$ и $1.5 \mathrm{~s})$ и при различных значениях $z(0.6,0.7$ и $0.8 \mathrm{~mm})$. В результате проведенного численного моделирования наблюдалось визуальное совпадение температурных профилей, соответствующих наконечникам с толщинами 0.02 и $0.2 \mathrm{~mm}$. Это объясняется тем, что при увеличении объема наконечника хоть и происходит уменьшение интенсивности источника на единицу объема, но суммарная интенсивность источника по всему наконечнику остается неизменной (см. уравнения (1) и (2)). Таким образом, при проведении численных расчетов, вне зависимости от реальных размеров наконечника, можно использовать толщину карбонизированного слоя, равную $0.2 \mathrm{~mm}$.

\section{Алгоритм решения задачи оптимального управления и его численная реализация}

Пусть для коэффициента теплопроводности справедливо представление (6). Ввиду нелинейности задачи, для нахождения оптимальной пары $P_{\varphi}, P_{\theta}$, минимизирующей функцию $J_{0}$, будем использовать следующий итерационный алгоритм.

0. Инициализируем счетчик итерационного процесса, $n=0$.

1. Задаем начальное управление $\left(P_{\varphi}^{(0)}, P_{\theta}^{(0)}\right)$.

2. Увеличиваем счетчик итерационного процесса, $n=n+1$.

3. Используя $\left(P_{\varphi}^{(n-1)}, P_{\theta}^{(n-1)}\right)$ в качестве аппроксимации пары $\left(P_{\varphi}, P_{\theta}\right)$, из $(10)-(13)$ находим функции $\theta_{1}, \theta_{2}, \theta_{3}$ и по ним соответствующие коэффициенты $C_{i}$, $i=1,2, \ldots, 5$.

4. Из равенств (15), (16) (или (17), (18) в случае фиксированной черноты наконечника) находим управление $\left(P_{\varphi}^{(n)}, P_{\theta}^{(n)}\right)$.

5. Переходим к шагу 2.

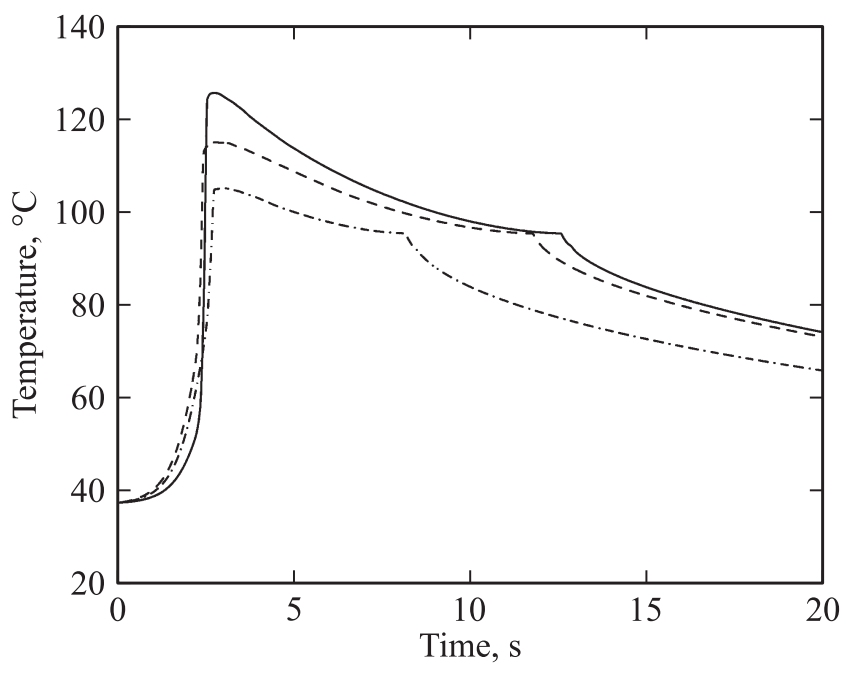

Рис. 5. Сходимость итерационного алгоритма решения задачи оптимального управления: сплошная линия - температурный профиль, соответствующий $P=\left(P_{\varphi}, P_{\theta}\right)=(7,3)$, который требуется приблизить; штрихпунктирная линия - температурный профиль, соответствующий начальному приближению $P^{(0)}=(12,0)$; штриховая линия - температурный профиль, полученный после первой итерации, $P^{(1)}=(14.99,0.05)$.

При реализации итерационного алгоритма, следуя $[12-14]$, на каждом $n$-м шаге при нахождении $C_{i}$, $i=1,2, \ldots, 5$, будем использовать следующее представление для коэффициента теплопроводности крови:

$k(x, t)= \begin{cases}k_{0}, & \text { если } P_{\varphi}^{(n-1)} \theta_{1}+P_{\theta}^{(n-1)} \theta_{2}+\theta_{3}<95, \\ 200 k_{0}, & \text { если } P_{\varphi}^{(n-1)} \theta_{1}+P_{\theta}^{(n-1)} \theta_{2}+\theta_{3} \geq 95,\end{cases}$

где $k_{0}$ - значение коэффициента теплопроводности крови, соответствующее температуре в начальный момент времени.

Возьмем в качестве заданной функции $\theta_{d}$, которую требуется приблизить с помощью представленного выше итерационного алгоритма, температурный профиль в точке $(1.5,10)$, найденный путем численного решения задачи $(1)-(5)$ при $\left(P_{\varphi}, P_{\theta}\right)=(7,3)$ (рис. 2). Такой выбор функции $\theta_{d}$ сделан для иллюстрации работы алгоритма и позволяет заранее знать управление $\left(P_{\varphi}, P_{\theta}\right)$, обеспечивающее минимум функции $J_{0}$. Это дает возможность проанализировать сходимость к нему итерационного алгоритма.

В качестве начального приближения $P_{\varphi}^{(0)}$ и $P_{\theta}^{(0)}$ возьмем значения 12 и 0. После первой итерации получаем приближение управления $\left(P_{\varphi}^{(1)}, P_{\theta}^{(1)}\right)=(14.99,0.05)$. Температурные профили, соответствующие нулевому и первому приближению, представлены на рис. 5. После второй итерации получаем приближение управления $\left(P_{\varphi}^{(2)}, P_{\theta}^{(2)}\right)=(8.01,2.51)$, соответствующий температурный профиль представлен на рис. 6. После четвертой итерации полученное приближение температурного профиля визуально неразличимо с 


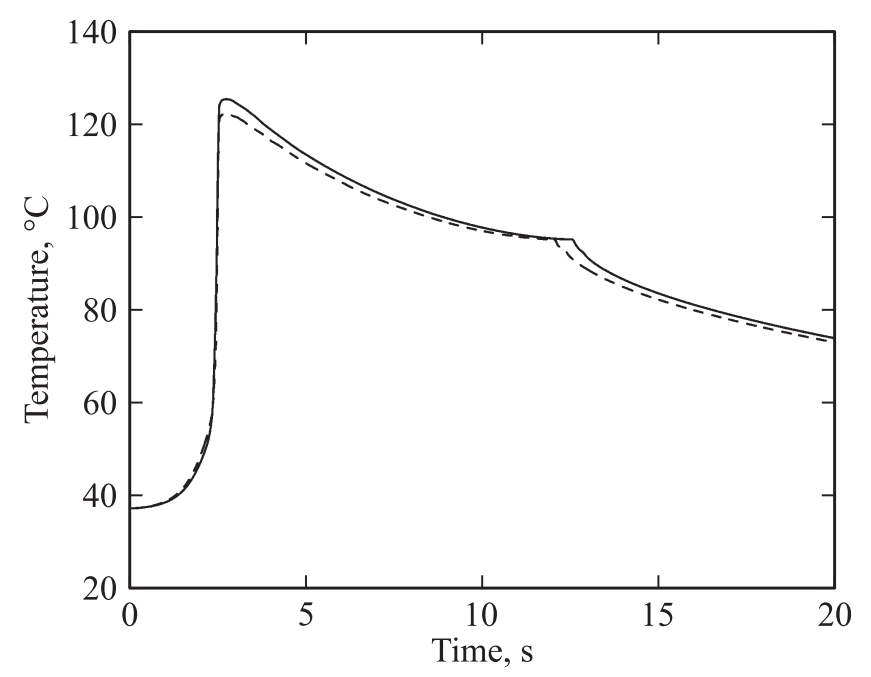

Рис. 6. Сходимость итерационного алгоритма решения задачи оптимального управления: сплошная линия - температурный профиль, соответствующий $P=\left(P_{\varphi}, P_{\theta}\right)=(7,3)$, который требуется приблизить; штриховая линия - температурный профиль, полученный после двух итераций, $P^{(2)}=(8.01,2.51)$. График, полученный после четырех итераций и соответствующий $P^{(4)}=(6.75,3.07)$, визуально неразличим со сплошной линией.

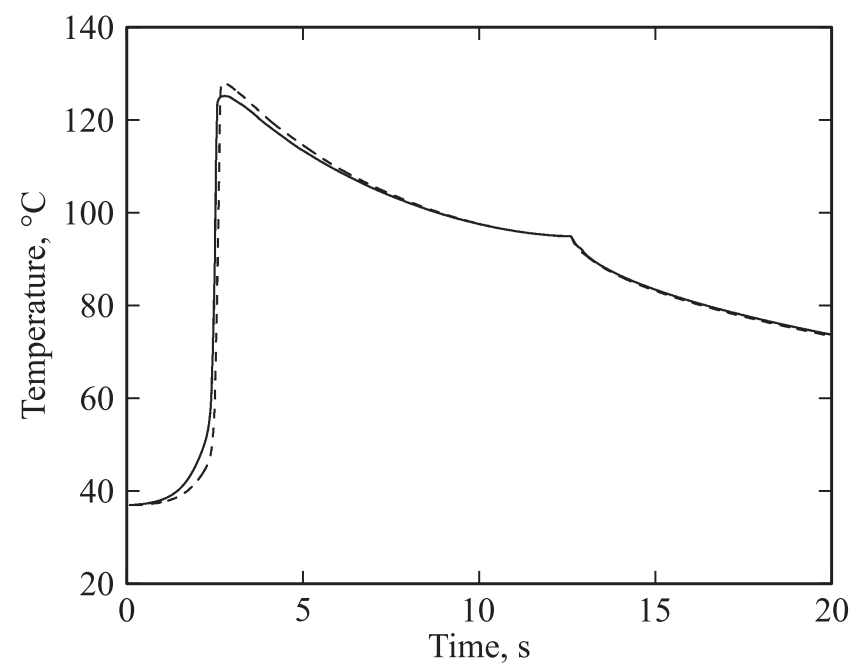

Рис. 7. Решение задачи оптимального управления при фиксированной черноте карбонизированного слоя $\sigma=0.5$ : сплошная линия - температурный профиль, соответствующий $P=\left(P_{\varphi}, P_{\theta}\right)=(7,3)$, который требуется приблизить; штриховая линия - температурный профиль, полученный по формулам (17), (18) после трех итераций, $P^{(3)}=(3.97,3.97)$.

функцией $\theta_{d}$, соответствующее значение управления $\left(P_{\varphi}^{(4)}, P_{\theta}^{(4)}\right)=(6.75,3.07)$. На основе представленного эксперимента можно сделать вывод о быстрой сходимости итерационного алгоритма.

Целью следующего эксперимента было получить приближение функции $\theta_{d}$ при фиксированной черноте наконечника. С практической точки зрения это означает, что не требуется, используя специальные технологии, подгонять черноту наконечника под значение, полученное с помощью формул (15) и (16). Можно измерить черноту наконечника имеющегося оптоволокна и затем с помощью формул (17) и (18) определить оптимальную мощность, которая будет обеспечивать наилучшее приближение. Как и ранее, заданная функция $\theta_{d}$ соответствует $\left(P_{\varphi}, P_{\theta}\right)=(7,3)$, что соответствует мощности лазера $10 \mathrm{~W}$ и черноте наконечника $\sigma=0.3$. Пусть имеется оптоволокно с чернотой наконечника $\sigma=0.5$. Требуется подобрать суммарную мощность $P_{\varphi}+P_{\theta}$, чтобы приблизить решение задачи $(1)-(5)$ к функци $\theta_{d}$. После трех итераций алгоритма с использованием формул (17) и (18) получено достаточно хорошее приближение функции $\theta_{d}$ (рис. 7), соответствующее управлению $\left(P_{\varphi}^{(3)}, P_{\theta}^{(3)}\right)=(3.97,3.97)$. То есть при изменении черноты наконечика с 0.3 до 0.5 , возникшего, например, в результате эксплуатации оптоволокна [18], для достижения хорошего приближения функции $\theta_{d}$ достаточно уменьшить мощность лазера с 10 до $8 \mathrm{~W}$.

При реализации итерационной процедуры на каждом временном шаге функции $\theta_{1}, \theta_{2}$ и $\theta_{3}$ находились с помощью метода конечных элементов с использованием пакета FreeFEM++ [24].

\section{Заключение}

В работе предложен и реализован итерационный алгоритм, определяющий оптимальные параметры излучения (мощности источника, идущие на излучение и на нагрев наконечника оптоволокна) при проведении процедуры ВВЛА для получения наилучшего приближения к заданному температурному профилю в некоторой точке модельной области. Предложенный алгоритм обладает быстрой сходимостью и обеспечивает хорошую аппроксимацию. Направления дальнейших исследований авторов будут связаны с обобщением алгоритма на случай, позволяющий аппроксимировать требуемый температурный профиль на внутренней стенке вены для обеспечения облитерации сосуда и одновременно гарантировать безопасную температуру в перивенозной ткани.

\section{Финансирование работы}

Исследование выполнено при финансовой поддержке РФФИ в рамках научного проекта № 20-01-00113 (а).

\section{Конфликт интересов}

Авторы заявляют, что у них нет конфликта интересов.

\section{Список литературы}

[1] Boné S.C. // Rev. Patol. Vasc. 1999. V. 5. P. 35.

[2] Van den Bos R.R., Neumann M., De Roos K.-P., Nijsten T. // Dermatol. Surg. 2009. V. 35. N 8. P. 1206. 
[3] Ahadiat O., Higgins S., Ly A., Nazemi A., Wysong A. // Dermatol. Surg. 2018. V. 44. N 5. P. 679.

[4] Malskat W.S.J., Stokbroekx M.A.L., Van der Geld C.W.M., Nijsten T.E.C., Van den Bos R.R. // Lasers Med. Sci. 2014. V. 29. P. 423.

[5] Красников И.В., Сетейкин А.Ю., Попов А.П. // Опт. и спектр. 2015. Т. 118. № 4. С. 698; Krasnikov I.V., Seteikin A.Y., Popov A.P. // Opt. Spectrosc. 2015. V. 118. N 4. P. 668.

[6] Прохоров И.В., Яровенко И.П. // Опт. и спектр. 2006. T. 101. № 5. C. 817; Prokhorov I.V., Yarovenko I.P // Opt. Spectrosc. 2006. V. 101. N 5. P. 769.

[7] Яровенко И.П., Прохоров И.В. // Опт. и спектр. 2018. T. 124. № 4. C. 534; Yarovenko I.P., Prokhorov I.V. // Opt. Spectrosc. 2018. V. 124. N 4. P. 567.

[8] Сетейкин А.Ю. // Опт. и спектр. 2005. Т. 99. № 4. С. 685; Seteikin A.Y. // Opt. Spectrosc. 2005. V. 99. N 4. P. 659.

[9] Ковтанюк А.Е., Гренкин Г.В., Чеботарев А.Ю. // Опт. и спектр. 2017. Т. 123. № 2. С. 194; Kovtanyuk A.E., Grenkin G.V., Chebotarev A.Y. // Opt. Spectrosc. 2017. V. 123. N 2. P. 205.

[10] Chebotarev A.Y., Grenkin G.V., Kovtanyuk A.E., Botkin N.D., Hoffmann K.-H. // Commun. Nonlinear Sci. Numer. Simul. 2018. V. 57. P. 290.

[11] De Boer A., Oliveira J.L.G., Van der Geld C.W.M., Malskat W.S.J., Van den Bos R., Nijsten T., Van Gemer M.J.C. // Int. J. Heat and Mass Transfer. 2017. V. 113. P. 153.

[12] Van Ruijven P.W.M., Poluektova A.A., Van Gemert M.J.C., Neumann H.A.M., Nijsten T., Van der Geld C.W.M. // Lasers Med. Sci. 2014. V. 29. P. 431.

[13] Poluektova A.A., Malskat W.S.J., Van Gemert M.J.C., Vuylsteke M.E., Bruijninckx C.M.A., Neumann H.A.M., Van der Geld C.W.M. // Lasers Med. Sci. 2014. V. 29. P. 441.

[14] Malskat W.S.J., Poluektova A.A., Van der Geld C.W.M., Neumann H.A.M., Weiss R.A., Bruijninckx C.M.A., Van Gemert M.J.C. // Lasers Med. Sci. 2014. V. 29. P. 393.

[15] Mordon S., Wassmer B., Zemmouri J. // BioMedical Engineering OnLine. 2006. V. 5. N. art. 26.

[16] Mordon S., Wassmer B., Zemmouri J. // Lasers Surg. Med. 2007. V. 39. P. 256.

[17] Marshak R. // Phys. Rev. 1947. V. 71. N 7. P. 443.

[18] Amzayyb M., Van den Bos R.R., Kodach V.M., De Bruin D.M., Nijsten T., Neumann H.A.M., Van Gemert M.J.C. // Lasers Med. Sci. 2010. V. 25. P. 439.

[19] Yusupov V.I., Chudnovskii V.M., Bagratashvili V.N. // Laser Physics. 2010. V. 20. N 7. P. 1641.

[20] Жигарьков В.С., Юсупов В.И., Цыпина С.И., Баграташвили В.Н. // Квант. электрон. 2017. Т. 47. № 10. С. 942; Zhigarkov V.S., Yusupov V.I., Tsypina S.I., Bagratashvili V.N. // Quant. Electroni. 2017. V. 47. N 10. P. 942.

[21] Беликов А.В., Скрипник А.В., Курнымев В.Ю., Шатилова К.В. // Квант. электрон. 2016. Т. 46. № 6. С. 534; Belikov A.V., Skrypnik A.V., Kurnyshev V.Yu., Shatilova K.V. // Quant. Electron. 2016. V. 46. N 6. P. 534.

[22] Беликов А.В., Скрипник А.В. // Квант. электрон. 2020. T. 50. № 2. C. 95; Belikov A.V., Skrypnik A.V. // Quant. Electron. 2020. V. 50. N 2. P. 95.

[23] Bredikhin V.I. // Mater. Res. Express. 2019. V. 6. N. art. 065412.

[24] Hecht F. // J. Numer. Math. 2012. V. 20. N 3-4. P. 251. 